\title{
Russell Merris
}

The Laplacian permanental polynomial for trees

Czechoslovak Mathematical Journal, Vol. 32 (1982), No. 3, 397-403

Persistent URL: http://dml.cz/dmlcz/101816

\section{Terms of use:}

(C) Institute of Mathematics AS CR, 1982

Institute of Mathematics of the Czech Academy of Sciences provides access to digitized documents strictly for personal use. Each copy of any part of this document must contain these Terms of use.

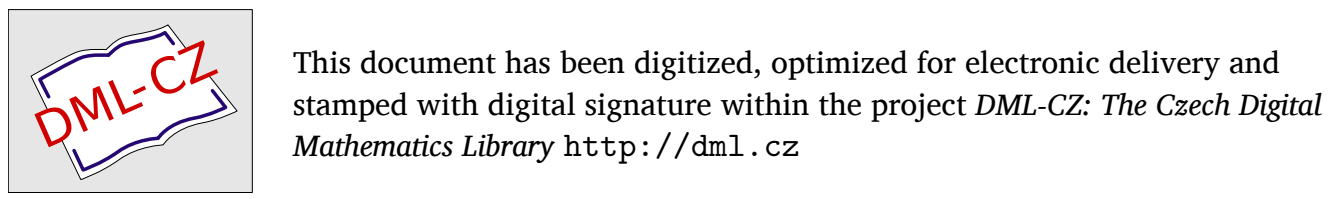




\title{
THE LAPLACIAN PERMANENTAL POLYNOMIAL FOR TREES
}

\author{
RusSell Merris, Hayward*)
}

(Received March 3, 1981)

\section{INTRODUCTION}

Let $G=(V, E)$ be a graph without loops or multiple edges, with vertex set $V=$ $=\left\{v_{1}, v_{2}, \ldots, v_{n}\right\}$, and with edge set $E$. We regard the edges of $G$ as two-element subsets of $V$. The adjacency matrix $A(G)$ afforded by $G$ is an $n$ by $n$ matrix with zeros on the main diagonal. When $i \neq j$, the $(i, j)$ entry of $A(G)$ is 1 if $\left\{v_{i}, v_{j}\right\} \in E$ and 0 , otherwise. Clearly, $A(G)$ depends not only on $G$ but on the ordering of $V$. It is easy to see, however, that the effect of permuting the elements of $V$ is to impose a (corresponding) permutation similarity on $A(G)$. In fact, more is true, namely, $G_{1}$ and $G_{2}$ are isomorphic graphs if and only if $A\left(G_{1}\right)$ is permutation similar to $A\left(G_{2}\right)$. Thus, any function of $A(G)$ which is preserved under permutation similarity is a function of the underlying graph. The characteristic polynomial is such a function. Indeed, the hope was briefly entertained that the characteristic polynomial of $A(G)$ characterizes $G$. We call two graphs adjacency cospectral if their adjacency matrices afford the same characteristic polynomial. Numerous examples of nonisomorphic, adjacency cospectral graphs are now known. Perhaps the most triumphant result along these lines is due to A. J. Schwenk. He proved that if $t_{n}$ is the number of (unlabeled) trees on $n$ vertices and if $s_{n}$ is the number of such trees which are not adjacency cospectral with a nonisomorphic tree, then $\lim _{n \rightarrow \infty}\left(s_{n} / t_{n}\right)=0$. One reason for the failure of the characteristic polynomial to distinguish graphs may be that it is preserved under arbitrary similarities. A function generally preserved only under permutation similarities might be more suitable. The permanent of the characteristic matrix (henceforth the permanental polynomial) of $A(G)$ is such a function. However, it is proved in [7] that, at least for trees, the permanental polynomial of $A(G)$ distinguishes nothing which was not already distinguished by the characteristic polynomial. In particular, Schwenk's Theorem remains valid if "characteristic polynomial" is replaced with "permanental polynomial".

Another suggestion is that $A(G)$ is just the wrong matrix to look at. Consisting only of zeros and ones, it is relatively easy to deal with. On the other hand, such a simple

*) The research leading to this article was supported by NSF grant MCS 77-28437. 
matrix may be expected to lead more easily to "algebraic accidents". Following W. N. Anderson and T. D. Morley, we are led to consider the Laplacian matrix $L(G)=D(G)-A(G)$, where $D(G)$ is the diagonal matrix whose $(i, i)$ entry is the valence (or degree) of vertex $v_{i}, 1 \leqq i \leqq n$. The Laplacian matrix (also called the "matrix of admittance" [3, p. 27]) has some advantages over the Adjacency matrix, in spite of presenting greater computational difficulties. (For example, $L(G)$ is positive semidefinite, a property exploited by M. Fiedler [5] in his work on algebraic connectivity of graphs.) Moreover, it remains true that $G_{1}$ is isomorphic to $G_{2}$ if and only if $L\left(G_{1}\right)$ is permutation similar to $L\left(G_{2}\right)$. We wonder how well the permanental polynomial of the Laplacian matrix distinguishes graphs. Following the success of Schwenk, we might look for examples of nonisomorphic but "Laplacian copermanental" graphs among the trees. It is the purpose of this paper to present a graph theoretical characterization of the coefficients of per $(x I-L(T))$ for trees $T$.

\section{RESULT}

For any graph $G=(V, E)$ on $n$ vertices, let and

$$
\operatorname{det}(x I-L(G))=x^{n}-d_{1}(G) x^{n-1}+d_{2}(G) x^{n-2}-\ldots+(-1)^{n} d_{n}(G),
$$

$$
\operatorname{per}(x I-L(G))=x^{n}-p_{1}(G) x^{n-1}+p_{2}(G) x^{n-2}-\ldots+(-1)^{n} p_{n}(G) .
$$

Denote by $Q_{k, n}$ the set of strictly increasing functions from $\langle k\rangle=\{1,2, \ldots, k\}$ into $\langle n\rangle$. If $\alpha, \beta \in Q_{k, n}$, denote by $L(G)[\alpha \mid \beta]$ the $k$ by $k$ submatrix of $L(G)$ lying in the rows $\alpha$ and columns $\beta$, i.e., the $k$ by $k$ matrix whose $(i, j)$ entry is the $(\alpha(i), \beta(j))$ entry of $L(G)$. Then it is immediate from (1) and (2) that

and

$$
d_{k}(G)=\sum_{\alpha \in Q_{k}, n} \operatorname{det}(L(G)[\alpha \mid \alpha])
$$

$$
p_{k}(G)=\sum_{\alpha \in Q_{k}, n} \operatorname{per}(L(G)[\alpha \mid \alpha]) .
$$

In particular, $d_{1}(G)=p_{1}(G)=\operatorname{trace} L(G)=\sum$ vertex valencies $=2 m$, where $m$ is the number of edges of $G$. Moreover, $d_{n}(G)=\operatorname{det} L(G)$ and $p_{n}(G)=\operatorname{per} L(G)$.

A graph theoretical interpretation for $d_{k}(G)$ was given in [4] (also described in $\left[2\right.$, p. 44] and [3, p. 38]), namely $d_{k}(G)=\sum g(F), k=1,2, \ldots, n$, where the summation is over all edge subgraphs $F$ of $G$ which have $k$ edges and are forests, and $g(F)$ is the product of the numbers of vertices in the connected components of $F$. We are able to give a vaguely analogous interpretation of $p_{k}(G)$ in case $G$ is a tree. Before presenting this interpretation we are obliged to introduce some notation.

Let $[V, E]=\{(v, e) \in V \times E: v \in e\}$. It might be helpful to think of the elements of $[V, E]$ as "half-edges" or "marimba sticks". Two ordered pairs $\left(v_{1}, e_{1}\right)$ and $\left(v_{2}, e_{2}\right)$ of $[V, E]$ will be said to overlap if $v_{1}=v_{2}$ or $e_{1}=e_{2}$. A distinguished subset of 
$[V, E]$ is one which consists of pairwise nonoverlapping elements. If $z \in E$, let

$$
[V \backslash z, E]=\{(v, e) \in[V, E]: v \notin z\} .
$$

Finally, for $t=1,2, \ldots,[k / 2]$, where $[k / 2]$ is the greatest integer in $k / 2$, define $c_{k, t}(G)$ to be the summation, over all collections $z_{1}, z_{2}, \ldots, z_{t} \in E$ of $t$ pairwise disjoint edges of $G$, of the total number of $(k-2 t)$-element distinguished subsets of $\bigcap_{j=1}^{t}\left[V \backslash z_{j}, E\right]$. (If $t=k / 2$, then $c_{k, t}(G)$ is simply the number of collections of $(k / 2)$ pairwise disjoint edges of $G$.)

Theorem. Let $T$ be a tree. Then

$$
p_{k}(T)=d_{k}(T)+\sum_{t=1}^{[k / 2]} 2^{t} c_{k, t}(T) .
$$

(Since $L(T)$ is positive semidefinite symmetric, $p_{k}(T) \geqq d_{k}(T)$ by Schur's Inequality. Equation (5) shows what has been discarded to obtain the inequality.)

Example. Let $G$ be the graph

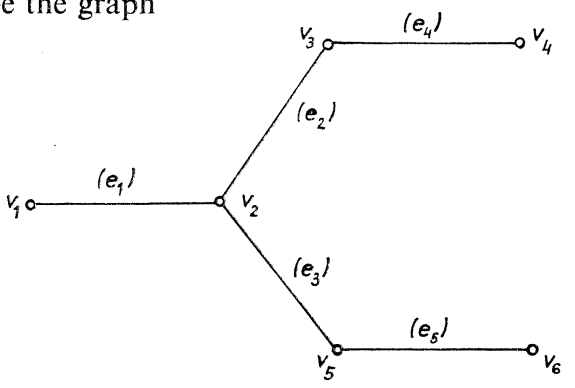

Then, abbreviating $\left(v_{i}, e_{j}\right)$ with $i j,[V, E]=\{11,21,22,23,32,34,44,53,55,65\}$. We calculate $p_{6}(G)=$ per $L(G)$ using the Theorem. Since the rows of $L(G)$ sum to zero, $d_{6}(G)=\operatorname{det} L(G)=0$. Observe

$$
c_{6,1}(G)=\sum_{z \in E} \text { number of 4-element distinguished subsets of }[V \backslash z, E] .
$$

If $e_{1}=\left\{v_{1}, v_{2}\right\}, \quad\left[V \backslash e_{1}, E\right]=\{32,34,44,53,55,65\}$. The only pairwise nonoverlapping (i.e., distinguished) 4-element subset of $\left[V \backslash e_{1}, E\right]$ is $\{32,44,53,65\}$. Each of the other four edges gives rise to exactly one 4-element distinguished subset:

$$
\begin{aligned}
& e_{2}=\left\{v_{2}, v_{3}\right\} \rightarrow\{11,44,53,65\}, \\
& e_{3}=\left\{v_{2}, v_{5}\right\} \rightarrow\{11,32,44,65\}, \\
& e_{4}=\left\{v_{3}, v_{4}\right\} \rightarrow\{11,22,53,65\}, \\
& e_{5}=\left\{v_{5}, v_{6}\right\} \rightarrow\{11,23,32,44\} .
\end{aligned}
$$

Thus, $c_{6,1}(G)=5$. Letting $t=2$,

$$
c_{6,2}(G)=\sum_{z_{1}, z_{2} \in E} \sum_{z_{1} \cap z_{2}=\emptyset} \text { number of 2-element distinguished subsets of }
$$


Consider the disjoint edges $e_{4}$ and $e_{5}$. Then $\left[V \backslash e_{4}, E\right]=\{11,21,22,23,53,55,65\}$ and $\left[V \backslash e_{5}, E\right]=\{11,21,22,23,32,34,44\}$. The intersection of these two sets is $\{11,21,22,23\}$. We are looking for all possible choices of 2 nonoverlapping elements from this set. There are two, namely $\{11,22\}$ and $\{11,23\}$. Each of the other pairs of disjoint edges gives rise to one 2-element distinguished subset, explicitly,

$$
\begin{array}{ll}
e_{1} \text { and } e_{4} \rightarrow\{53,65\}, \\
e_{1} \text { and } e_{5} \rightarrow\{32,44\}, \\
e_{2} \text { and } e_{5} \rightarrow\{11,44\}, \\
e_{3} \text { and } e_{4} \rightarrow\{11,65\} .
\end{array}
$$

These four, together with the two already obtained (for $e_{4}$ and $e_{5}$ ) yield $c_{6,2}(G)=6$. Finally, letting $t=3$,

$$
\begin{gathered}
c_{6,3}(G)=\sum_{z_{1}, z_{2}, z_{3} \in E} 1 . \\
\text { pairwise disjoint }
\end{gathered}
$$

Since there is only one triple of pairwise disjoint edges in $G$, namely $e_{1}, e_{4}$ and $e_{5}$, $c_{6,3}(G)=1$. Putting this information into (5) gives

$$
\begin{aligned}
p_{6}(G) & =0+2 c_{6,1}(G),+4 c_{6,2}(G)+8 c_{6,3}(G)= \\
& =0+2(5)+4(6)+8(1)= \\
& =42 .
\end{aligned}
$$

As an application of the Theorem, we affirm a conjecture made in [7]. The star graph on $n$ vertices is the tree with one vertex of valence $n-1$ and the remaining $n-1$ vertices each of valence 1 . We can now state the conjecture as a fact.

Corollary. If $G$ is a connected graph on $n$ vertices, then per $L(G) \geqq 2(n-1)$ with equality if and only if $G$ is the star graph.

Proof. It was established in Theorem 3.2 of [7] that if $G_{1}$ is a proper subgraph of the connected graph $G$, then per $L(G)>$ per $L\left(G_{1}\right)$. Since the trees are the minimally connected graphs on $n$ vertices, it suffices to prove the result for trees. We appeal to Equation (5) with $k=n$. As we argued in the Example, $d_{k}(T)=\operatorname{det} L(T)=0$. Consider the case $t=1$. Let $z$ be an edge of $T$. We seek the number of $(n-2)$ element distinguished subsets of $[V \backslash z, E]$. After removing the edge $z$ and the vertices at either end from $T$, we obtain a "pruned" tree with $n-2$ vertices, some edges, and some "dangling half-edges". Now, each of the $n-2$ remaining vertices must be represented (exactly once) as the first member of an ordered pair in any distinguished subset. Therefore, each of the end vertices in the pruned tree must appear with its associated edge. Removing these ordered pairs (pears?) from the pruned tree leaves new, pseudo end vertices. Continuing in this way, we find there to be exactly one distinguished subset of $[V \backslash z, E]$ for each edge $z$ of $T$. Thus, $c_{n, 1}(T)=$ 
$=m=(n-1)$ for any tree $T$. If $T_{0}$ is the star, there are no two disjoint edges, much less $t$-tuples of disjoint edges for $t>2$. Thus, per $L\left(T_{0}\right)=2(n-1)$. If $T$ is a tree on $n$ vertices which is not the star, then $T$ has two vertices of valence at least 2 , say $v_{1}$ and $v_{2}$. Let $z_{1}$ and $z_{2}$ be disjoint edges which adjoin $v_{1}$ and $v_{2}$, respectively. A slight modification of the argument above yields the existence of a distinguished subset of $\left[V \backslash z_{1}, E\right] \cap\left[V \backslash z_{2}, E\right]$. Thus, $c_{n, 2}(T)>0$. It follows that per $L(T) \geqq$ $\geqq \operatorname{per} L\left(T_{0}\right)+4$ for any tree $T \neq T_{0}$.

\section{PROOF OF THEOREM}

In outline, our proof follows the corresponding one for the characteristic polynomial to be found in [2]. The crucial step is an analog of a lemma of H. Poincaré.

Consider a graph $G=(V, E)$ with $n$ vertices and $m$ edges. For each edge $e_{t}=$ $=\left\{v_{i}, v_{j}\right\}$ of $G$, choose (arbitrarily) one of $v_{i}, v_{j}$ to be the positive end of $e_{t}$, and the other to be the negative end. The incidence matrix $N(G)$, with respect to the resulting directed graph, is an $n$ by $m$ matrix, the $(i, j)$ entry of which is +1 if $v_{i}$ is the positive and of $e_{j},-1$ if $v_{i}$ is the negative end of $e_{j}$, and 0 otherwise. Each column of $N(G)$ contains exactly one +1 and exactly one -1 . Moreover, $N(G) N(G)^{t}=L(G)$.

Next, we denote by $G_{k, m}$ the set of nondecreasing functions from $\langle k\rangle$ into $\langle m\rangle$. For $\alpha \in Q_{k, n}$ and $\gamma \in G_{k, m}, N(G)[\alpha \mid \gamma]$ denotes the $k$ by $k$ matrix whose $(i, j)$ entry is the $(\alpha(i), \gamma(j))$ entry of $N(G)$. Since $\gamma$ need not be one-to-one, $N(G)[\alpha \mid \gamma]$ may not be a submatrix of $N(G)$. Some of the columns may be repeated. Regarding $\gamma$ as a sequence of integers of length $k$ chosen (with repetitions permitted) from $\langle m\rangle$, let $v(\gamma)$ be the product of the factorials of the multiplicities of the distinct integers appearing in the sequence $\gamma$. (If, e.g., $k=6, m=7, \gamma=(1,1,1,4,5,5)$, then $v(\gamma)=$ $=6.2=12$.) We are now ready to invoke the Binet-Cauchy Theorem for permanents $\left[6\right.$, p. 20]: If $\alpha \in Q_{k, n}$. then

$$
\operatorname{per}(L(G)[\alpha \mid \alpha])=\sum_{\gamma \in G_{k}, m} \frac{1}{v(\gamma)} \operatorname{per}(N(G)[\alpha \mid \gamma]) \operatorname{per}\left(N(G)^{t}[\gamma \mid \alpha]\right) \text {. }
$$

Since $N(G)^{t}[\gamma \mid \alpha]=(N(G)[\alpha \mid \gamma])^{t}$, and since the permanent is invariant under the transpose operation, we obtain

$$
\operatorname{per}(L(G)[\alpha \mid \alpha])=\sum_{\gamma \in G_{k}, m} \frac{1}{v(\gamma)}(\operatorname{per}(N(G)[\alpha \mid \gamma]))^{2} .
$$

Lemma. Let $T$ be a tree with $n$ vertices and $m(=n-1)$ edges. If $1 \leqq k \leqq m$, then $\operatorname{per}(N(T)[\alpha \mid \beta])= \pm \operatorname{det}(N(T)[\alpha \mid \beta])$, for all $\alpha \in Q_{k, n}$ and $\beta \in Q_{k, m}$. In particular, any square submatrix of an incidence matrix of a tree has permanent equal to $0,+1$, or -1 .

Proof. Let $S$ be a $k$ by $k$ submatrix of $N(T)$. Each column of $S$ contains at most 
two nonzero entries. If each column of $S$ were to contain exactly two nonzero entries, then $S$ would be an incidence matrix for an edge-subgraph of $T$. In particular, $S$ would be an incidence matrix of a forest. But this is impossible because $S$ is square, yet the number of edges of a forest is strictly less than the number of vertices. (Specifically, edges = vertices-components.) We may assume, therefore, that there is a column of $S$ containing at most one nonzero entry. Of course, if there is a zero column of $S$, then det $S=$ per $S=0$. Otherwise, suppose column $j$ contains exactly one nonzero entry in row $i$. Then per $S= \pm \operatorname{per} S(i \mid j)$ and $\operatorname{det} S= \pm \operatorname{det} S(i \mid j)$, where $S(i \mid j)$ is the submatrix of $S$ obtained by deleting row $i$ and column $j$. Now, $S(i \mid j)$ is a $(k-1)$ by $(k-1)$ submatrix of $N(T)$. As before, either $S(i \mid j)$ contains a coiumn of zeros or a column with exactly one nonzero entry. Continue the process of reduction of the size of the submatrix until a column of zeros appears or a 1 by 1 submatrix is reached. In either case, the result follows.

In [8], Poincaré proved that any square submatrix of an incidence matrix of a graph has determinant equal to $0,+1$, or -1 . It is easy to construct examples which show that the analogous result for permanent is false. It is for this reason that we have been able to obtain a theorem only for trees.

We now return to Equation (6). It follows from our Lemma, the Binet-Cauchy Theorem for determinants, Equations (3) and (4) that

$$
p_{k}(T)=d_{k}(T)+\sum_{\alpha \in Q_{k}, n} \sum \frac{1}{v(\gamma)}(\operatorname{per}(N(T)[\alpha \mid \gamma]))^{2},
$$

where the second summation is over those $\gamma \in G_{k, m}$ with $v(\gamma)>1$. Denote by $m_{r}(\gamma)$ the multiplicity of the integer $r$ in $\gamma$. (Then $v(\gamma)=\prod_{r=1}^{m}\left(m_{r}(\gamma) !\right)$.) If there is an integer $s$ such that $m_{s}(\gamma) \geqq 3$, then $N(T)[\alpha \mid \gamma]$ contains three equal columns. Since this repeated column contains at most two nonzero entries, it follows that $N(T)[\alpha \mid \gamma]$ has a 3 by $(k-2)$ submatrix of zeros. By the Frobenius-König Theorem, per $(N(T)$. . $[\alpha \mid \gamma])=0$ for such a $\gamma$ and any choice of $\alpha \in Q_{k, n}$. Thus, we may restrict the second summation in (7) to those $\gamma \in G_{k, m}$ satisfying $m_{r}(\gamma) \leqq 2, r=1,2, \ldots, m$, with at least one case of equality. If $m_{l}(\gamma)=2$, part of column $l$ of $N(T)$ occurs twice in $N(T)[\alpha \mid \gamma]$. If the nonzero entries in column $l$ of $N(T)$ lie in rows $i$ and $j$, then $N(T)[\alpha \mid \gamma]$ contains a 2 by $(k-1)$ submatrix of zeros (and hence per $(N(T)$. . $[\alpha \mid \gamma])=0$ ) unless both $i$ and $j$ are contained in (the sequence) $\alpha$. If $\alpha(p)=i$, $\alpha(q)=j, \gamma(r)=\gamma(s)=l$, then

$$
\operatorname{per}(N(T)[\alpha \mid \gamma])=-2 \operatorname{per}((N(T)[\alpha \mid \gamma])(p, q \mid r, s))
$$

where $(N(T)[\alpha \mid \gamma])(p, q \mid r, s)$ is the $(k-2)$ by $(k-2)$ submatrix of $N(T)[\alpha \mid \gamma]$ obtained by deleting rows $p$ and $q$, and columns $r$ and $s$.

Consider the case that $m_{r}(\gamma)=2$ for exactly $t$ values of $r$. Then $v(\gamma)=2^{t}$. Moreover, there are $t$ edges, $z_{1}, z_{2}, \ldots, z_{t}$ of $T$ such that the corresponding columns of $N(T)$ each occur twice in $N(T)[\alpha \mid \gamma]$. If two of these edges share a vertex, then $N(T)[\alpha \mid \gamma]$ 
has 4 columns with nonzero entries confined to (at most) 3 rows, i.e., $N(T)[\alpha \mid \gamma]$ contains a 4 by $(k-3)$ submatrix of zeros, and the corresponding permanent is zero. In case no two of $z_{1}, z_{2}, \ldots, z_{t}$ share a vertex, we may repeat the argument which led to (8) $t$-times to obtain $(-2)^{t}$ times the permanent of a $(k-2 t)$ by $(k-2 t)$ submatrix of $N(T)$. Appealing to the lemma, we obtain $(\operatorname{per}(N(T)[\alpha \mid \gamma]))^{2} / v(\gamma)=0$ or $2^{t}$. We summarize as follows: In the second summation in (7), from among those $\gamma \in G_{k, m}$ for which $m_{r}(\gamma)=2$ for exactly $t$ values of $r$ (and $m_{r}(\gamma)<2$ for the remaining values) we sum only over those $\gamma$ for which the repeated entries correspond to a collection of $t$ pairwise disjoint edges of $T$. For such a $\gamma,(\operatorname{per}(N(T)[\alpha \mid \gamma]))^{2} / v(\gamma)=2^{t}$ if and only if the corresponding $(k-2 t)$ by $(k-2 t)$ submatrix of $N(T)$ has permanent \pm 1 (i.e., not zero). This occurs if and only if $2 t$ of the entries of $\alpha$ correspond to the nonzero rows of the $t$ repeated columns of $\gamma$ and the remaining $(k-2 t)$ entries of $\alpha$ match up with the remaining $(k-2 t)$ entries of $\gamma$ to produce a $(k-2 t)$ by $(k-2 t)$ submatrix of $N(T)$ with a nonzero diagonal. In a $\gamma$ for which the $t$ repeated entries correspond to the pairwise disjoint edges $z_{1}, z_{2}, \ldots, z_{t}$, these conditions are equivalent to the existence of a distinguished subset of $\bigcap_{j=1}^{t}\left[V \backslash z_{j}, E\right]$. That this is so may easily be seen by visualizing the matrix $N(T)$ whose rows are indexed by vertices and whose columns are indexed by edges. Thus, $c_{k, t}(T)$ counts the number of occurrences of $2^{t}$ in the double summation on the right-hand side of (7).

\section{References}

[1] W. N. Anderson, Jr. and T. D. Morley: Eigenvalues of the Laplacian of a graph, TR 71-45, Univ. of Md., College Park, MD.

[2] N. Biggs: Algebraic Graph Theory, Cambridge Univ. Press, 1974.

[3] D. M. Cvetković, M. Doob and H. Sachs: Spectra of Graphs, Academic Press, New York, 1980.

[4] A. K. Kel'mans and V. M. Chelnokov: A certain polynomial of a graph and graphs with an extremal number of trees, J. Combinatorial Theory (B) 16 (1974), 197-214. Erratum, Ibid. 24 (1978), 375.

[5] M. Fiedler: Algebraic connectivity of graphs, Czech. Math. J. 23 (98) (1973), 298-305.

[6] M. Marcus and H. Minc: A Survey of Matrix Theory and Matrix Inequalities, Prindle, Weber and Schmidt, Boston, 1964.

[7] R. Merris, K. R. Rebman and W. Watkins: Permanental polynomials of graphs, Letters in Linear Algebra, Linear Algebra Appl. 38 (1981), 273-288.

[8] H. Poincaré: Second complément à l'analysis situs, Proc. London Math. Soc. 32 (1901), 277-308.

[9] I. Schur: Über endliche Gruppen und Hermitesche Formen, Math. Z. I (1918), 184-207.

[10] A. J. Schwenk: Almost all trees are cospectral, New Directions in Graph Theory (edited by F. Harary), Academic Press, 1973.

Author's address: California State University, Hayward, CA 94542, U.S.A. 